\title{
Plant speciation and polyploidy: in habitat divergence and environmental perspective
}

\author{
Umesh C. Lavania ${ }^{1}$ \\ Published online: 24 February 2020 \\ (c) Archana Sharma Foundation of Calcutta 2020
}

\begin{abstract}
Habitat divergence plays a driving role in speciation. Severe environmental fluactuations and/or stress may bring about unreduced gamete formation in germline or mitotic errors in somatic tissues, enabling the establishment of new genomic states. However, fixation of any such variation should involve merchanisms that produce reproductively isolated lineages. This could be realized either through polyploidy enabling alteration in genomic constitution involving chromosome duplication and/or numerical/structural chromome alterations or by hybridization/homoploidy. Whereas polyploidy may lead to stable genomic states owing to their tolerance to post-polyploid genetic modification; the interspecies hybridization is often maladaptive but can also promote speciation through generation of homoploid hybrids (i.e. hybridization without polyploidy) enabling reproductive isolation of evolving hybrids. However, homoploidy as a speciation mechanism is considered only as a minor contributor in evolution, but polyploidy, more particularly autopolyploidy stands apart as a major evolutionary force owing to its wide occurrence and short generation time, and shall continue to be as an effective speciation mechanism to sustain habitat disturbance emanating from rigors of climate change.
\end{abstract}

Keywords Polyploidy and speciation · Polyploidy and habitat divergence $\cdot$ Polyploidy and climate change $\cdot$ Polyploid fixation · Polyploidy and in vitro stress

\section{Polyploidy and speciation: contrasting viewpoints from 'evolutionary dead end'-to- 'major player in evolution'}

Despite widespread occurrence of polyploidy, it is often argued whether polyploidy is advantageous for evolutionary success $[20,24,25]$. There has been a long-standing debate over the evolutionary implications of genome doubling spanning the spectrum from: (1) polyploidy being an evolutionary dead end $[3,21,44]$ to (2) polyploidy being a major player with a significant role in evolution [18, 22, 35, 45].

Incidence of polyploidy is quite common in all groups of plants, but not so common in animals [25, 43]. Winkler [46] generated the first artificial polyploid, and is often credited with the first use of the term "polyploidy". Tremendous scientific efforts have undergone in polyploidy research,

Umesh C. Lavania

lavaniauc@yahoo.co.in

1 Departtment of Botany, University of Lucknow, Lucknow 226007, India including the publication of a treatises $[19,36]$, and special journal issues [2, 27, 39].

Stebbins [30] considered as an architect of the initial models of polyploid evolution with publications spanning over 70 years from 1929 to 1999 strongly influenced thinking about polyploidy for over 50 years and popularized five major themes as the central tenets of polyploid evolutionary thinking, suggesting that polyploidy is an "evolutionary dead end' with limited importance in diversification. $\mathrm{He}$ estimated that polyploids are formed in moderate frequency of $\sim 30-35 \%$, via single origin with limited evolutionary potential, most of which are allopolyploids and rarely as autopolyploids. The latter are considered as hinderance in evolution. Stebbins' strong views regarding the minor role of autopolyploidy in Nature had a huge impact, hindering research into this type of polyploidy for decades $[34,35]$. Levin $[16,17]$ challenged the then established models about polyploidy advanced by Stebbins, and emphasized that polyploidy has a significant role in generating novelty at a range of organizational levels and dynamic nature of polyploid genomes. Since then there has been resurgence of interest, 
and vast data have poured in, suggesting significant role of polyploidy in diversification and speciation.

In extensive discussion on occurrence of polyploidy [32, 35] two contrasing views are outlined: (1) Traditional view: polyploidy events are rare and considers most polyploids to be of single origin with low genetic diversity, (2) Current view: polyploidy invokes multiple origins and therefore evince high genetic diversity. The latter has led to paradigm change in our understanding about polyploidy in Evolution. It is now recognized that both allo- and autopolyploidy are extremely important in Nature [34, 35, 43].

\section{Polyploidy is a major player in evolution}

With the availability of new genetic tools of DNA based markers, In Situ hybridization, DNA sequencing, there has been tremendous resurgence in polyploidy research that have led to a new paradigm of polyploidy [13] leading to synthesis of series of topical reviews in top-notch journals, and publication of special issues of journals replacing the old thinking of polyploids considered as 'evolutionary dead end' -to- 'major player in evolution', suggesting that polyploid genomes are highly dynamic, beyond anything that Stebbins and his contemporaries could have predicted [34, 35, 43].

Genome sequencing exhibit evidence of ancient polyploidy events in all angiospers [7, 11]. Integration of comprehensive phylogenomic analysis of more than 12.6 million new ESTs from pivotal lineages and molecular time clock of land plant evolution led to identification of: (a) Two groups of ancestral Whole Genome Duplications (WGDs) - one in the common ancestor of extant seed plants and the other in the common ancestor of extant angiosperms, (b) Ancestral WGDs resulted in the diversification of regulatory genes important to seed and flower development, suggesting that they were involved in major innovations that ultimately contributed to the rise and eventual dominance of seed plants and angiosperms [11, 42].

Multiple rounds of WGD-polyploidy occurrence and diversification Recent investigations of entire genomes have shown that perhaps all eukaryotes possess genomes with considerable gene redundancy, much of which is the result of two or three rounds of ancient whole genome duplication events [4, 42, 44] Ancient, as well as more recent, polyploidy events have been documented in vertebrates, fungi, and ciliates, and occur extensively in green plants $[11,43]$. Multiple origins are now considered to be the rule in polyploid evolution. Nearly all polyploids that have been investigated with genetic markers show evidence of recurrent formation, whereby rapid changes post- polyploid formation occur in genome structure, gene content, gene expression, and methylation and other epigenetic regulators. Polyploidy itself has played a central role in shaping and restructuring plant genomes and has shattered earlier perceptions that polyploid species owe a genetically uniform genotype that represent evolutionary dead-ends; instead ancient polyploidy events are often associated with major clades [35, 37, 43].

Polyploidy is ubiquitous in green plants, with all angiosperms and all seed plants being of ancient polyploid origin Within plants, the incidence of polyploidy appears to be low or absent in liverworts, hornworts, cycads, and conifers, but is frequent in lycophytes, monilophytes, and angiosperms [10]. Wood et al. [47] estimated that of speciation events, $15 \%$ for flowering plants and $31 \%$ for ferns directly involve polyploidy.

\section{Autopolyploidy is quite common}

Because a major criterion for recognition of an autopolyploid has been polysomic inheritance, therefore detection of autopolyploids was long hampered by lack of easy tools for assessing genetic diversity and inheritance patterns. Studies on allozyme electrophoresis of diverse plants revealed a number of previously unrecognized autopolyploids, leading to more and more species being added to the list of natural autopolyploids [33, 35, 38]. Inference drawn on disomic versus polysomic inheritance from allozyme [40] and other genetic markers e.g., microsatellites $[12,31]$ have led to the revelation of a much higher incidence of natural autopolyploidy then thought earlier.

\section{Environmental constraints could elicit polyploidization}

Five mass extinctions of live forms are said to have happened owing to natural catestrophes over geological time scale with the loss and degradation habitat. However such crisis drove new ecological nitches enabling speciation and establishment of new species. Many flowering plants escaped fifth mass extinction occurring at the end of the Cretaceous by duplicating their genomes [8]. Two groups of ancestral Whole Genome Duplications (WGDs) evolved into terrestrial plant lineages - one in the common ancestor of seed plants and other in the common ancestor of extant angiosperms. Such ancestral WGDs are said to have led to diversification of regulatory genes contributing to rise and establishment of seed plants [11]. Alix et al. [1] have identified several critical genome replication/duplication events that have occurred during periods of major environmental and climate change. It is therefore surmised that genome duplication could be a force to escape/overcome the vagueries of harsh environment. This is consistent with the understanding that adaptive speciation is a survival strategy [5, 18]. This stems on account of broader adaptability and vast 
ecological tolerance [41] and higher invasive potential of diploids than their diploid relatives [26].

In this context, following arguments that favour higher incidence of increase in number of plant chromosome/polyploid formation under the stressful environment akin to climate change regime are worth considering:

(a) Chromosome variation and polyploidy realized during in vitro culture has evolutionary parallels in Nature

The biological environment excercised during in vitro culture of cells and tissues is not akin to natural conditions of growth and development, and is rather bestowed with stressful surroundings. The optimum nutritional culture medium made available during in vitro culture simply facilitates callus formation and subsequent growth. Therefore individual cell facing opportunity of growth in such environment is likely to face the developmental constrains and is likely to adopt measures to sustain its survival. One of the quick and easy recouse to survive/evolve could be genome duplication. In fact such an observation has been encountered during experimental studies while studying the pattern of chromosome change in in vitro culture with monthly passage of subculture $[14,15]$. It was observed that the diploid cells resort to genome multiplication during the course of subculture and stabilize at tetraploid level. The intermediate levels of genome multiplication that may accrue are selected against through morphogenetic sieve, leaving only genomically balanced euploid cells to sustain differentiation. This suggested that the biological stress inflicted in in vitro culture lead to polyploidization owing to mitotic errors [14]. The study of the pattern of chromosome variation during in vitro passage of subculture could be a simple means to study trends in chromosome evolution in Nature that may be happening over evolutionary time scale [15]. These observations underpin that genome multiplication could be a simple genomic strategy to evolve new forms with stressful environment that is likely to ensue with looming threats of climate change.

(b) Plant speciation in the age of climate change

Polyploid speciation is a long-term evolutionary survival strategy in plants consummated through generation of genetic variation to facilitate adaptation to new environments [5]. This is consummated through their enhanced physiological functions emnating from increase cell size and cell surface area. Unique morphological and novel traits in polyploids could increase their competitive ability supporting their survival in wider ecological niches and environmental constraints. Levin [18] opines that new polyploid species are likely to increase on account of their competitive adaptive advantage over diploid relatives, and incidence of occurrence of autopolyploids under the threats of climate change may be to the tune of $33 \%$. Such polyploids may competitively replace their ancestors, or may invade new territories. This is commensurrate with the episodes of genomic duplications that had occurred as an escape to environmental vaguries in the era of 5th mass extinction, leading to evolution of dominant terrestrial lineages [11].

(c) Autopolyploidy would be the common mode of speciation

Whereas genetic variation is an evolutionary and speciation strategy that go side by side with changing environment, but whole genome duplication remains a master survival strategy to gross environmental changes providing wider adaptively in shorter time. Higher incidence of polyploidy in the habitats facing high temperature fluctuations suggests that polyploidization is an intrinsic biological adaptation mechanism to withstand adverse landscapes [28]. The unreduced gamete formation shall quickly realize autopolyploidy in just two cycles, but for allopolyploidy that require integration of diverse genomes and duplication cycles it would be longer exercise and migration to new habitats. For homoploid hybrids even longer gestation may be required incurring hybridization in related but distinct species and subsequent establishment as reproductively isolated distinct lineages [23].

In the backdrop of above Levin [18] argues that autopolyploid speciation shall be the main type of plant speciation because production of unreduced gametes that facilitates autopolyploidization is positively correlated with nutrient, humidity, temperature variation and high levels of herbivory, a situation likely to be encountered under climate change regime. Allopolyploidy on account of greater ecological tolerance and greater invasive potential would be another form of speciation but its frequency of occurrence shall be second most important because it involves the additional step of participation of more than one species in its formation and subsequent stabilization, as well as formation of chromosomal rearrangements.

\section{Dominant plant speciation types}

Based on analysis of worldwide spatial distribution of polyploid frequencies, Rice et al. [29] have pinpointed that there is a clear latitudinal trend for increased occurrence of polyploidy away from equator. It is further highlighted that the attributes such as sufficient time (i.e., perenniality) and space (low species richness) facilitate establishment of new polyploid lineages to outcompete with pre-adapted diploid relatives, with proportionally higher increase in the herbaceous 
polyploid species, and most as perennials. While discussing the patterns of speciation vis-à-vis climate changes in the next 500 years, Levin [18] opines that if global climate undergoes major changes, then these major changes will inevitably lead to an increase in the number of plant chromosomes. This in effect could increase the current proportion of polyploids in angiosperms to 35-50\%, and an overall proportion up to $50 \%$ of the Earth's plant species as polyploids. He further argues that euploidization in herbaceous plants is 40-70 times more likely than aneuploidization, and evolution of short-statured herbaceous plants is more likely in response to major climatic changes in future.

Level of genetic divergence between the hybridizing species, preferably moderate diversity involving more than two species is likely to lead to the evolution of novel "adaptive systems" and promote speciation [6].

Gao [9] in his critical appraisal of the viewpoint advanced by Levin [18] has portrayed five main types of plant speciation events in proportionately decreasing order in the context of climate change. It is outlined that autopolyploid speciation would be most dominant type, followed by allopolyploid speciation. Speciation due to chromosomal rearrangements, homoploid hybrid speciation and lineage splitting shall remain the minor contributors.

Acknowledgements Support received from INSA, New Delhi as Senior Scientist is gratefully acknowledged.

\section{References}

1. Alix K, Gérard PR, Schwarzacher T, Heslop-Harrison JS. Polyploidy and interspecific hybridization: partners for adaptation, speciation and evolution in plants. Ann Bot. 2017;120:183-94.

2. Alix K, Schwarzacher T, Heslop-Harrison JS. (Guest Editors). Special issue on polyploidy in ecology and evolution. Special journal issue-Ann Bot. 2017; 120(2).

3. Arrigo N, Barker MS. Rarely successful polyploids and their legacy in plant genomes. Curr Opin Plant Biol. 2012;15:140-6.

4. Bowers JE, Chapman BA, Rong J, Paterson AH. Unravelling angiosperm genome evolution by phylogenetic analysis of chromosomal duplication events. Nature. 2003;422:433-8.

5. Cai L, Xi Z, Amorim M, et al. Widespread ancient whole-genome duplications in Malpighiales coincide with Eocene global climatic upheaval. New Phytol. 2019;221:565-76.

6. Comeault Aaron A, Matute Daniel R. Genetic divergence and the number of hybridizing species affect the path to homoploid hybrid speciation. Proc Natl Acad Sci USA. 2018;115:9761-6. https:// doi.org/10.1073/pnas.1809685115.

7. Comparative Genomics [CoGe] website. http://genomevolution. org $/ \mathrm{CoGe} /$

8. Fawcett JA, Maere S, Van de Peer Y. Plants with double genomes might have had a better chance to survive the Cretaceous-Tertiary extinction event. Proc Natl Acad Sci USA. 2009;106:5737-42.

9. Gao J. Dominant plant speciation types. A commentary on: 'plant speciation in the age of climate change'. Ann Bot. 2019;124:4-5. https://doi.org/10.1093/aob/mcz174.

10. Husband BC, Baldwin SJ, Suda J. The incidence of polyploidy in natural plant populations: major patterns and evolutionary processes. In: Greilhuber J, Doležel J, Wendel JF, editors. Plant genome diversity, vol. 2. Vienna: Springer; 2013. p. 255-76.

11. Jiao Y, Wickett NJ, Ayyampalayam S, et al. Ancestral polyploidy in seed plants and angiosperms. Nature. 2011;473:97-100.

12. Landergott UY, Naciri Y, Schneller JJ, Holderegger R. Allelic confi guration and polysomic inheritance of highly variable microsatellites in tetraploid gynodioecious Thymus praecox agg. Theor Appl Genet. 2006;113:453-65.

13. Lavania UC. Emerging trends in polyploidy research. Nucleus. 2015;58:1-2.

14. Lavania UC, Srivastava S. Ploidy dependence of chromosomal variation in callus cultures of Hyoscyamus muticus L. Protoplasma. 1988;145:55-8.

15. Lavania UC, Srivastava S. Evolutionary genomic change paralleled by differential responses of $2 \times$ and $4 \times$ calli cultures. Experientia. 1990;46:322-4.

16. Levin DA. Polyploidy and novelty in flowering plants. Am Nat. 1983;122:1-25.

17. Levin DA. The role of chromosomal change in plant evolution. New York: Oxford University Press; 2002.

18. Levin DA. Plant speciation in the age of climate change. Ann Bot. 2019;124:769-75. https://doi.org/10.1093/aob/mcz108.

19. Lewis WH, editor. Polyploidy. Biological relevance. New York: Plenum Press; 1980.

20. Madlung A. Polyploidy and its effect on evolutionary success: old questions revisited with new tools. Heredity. 2013;110:99-104. https://doi.org/10.1038/hdy.2012.79.

21. Mayrose I, Zhan SH, Rothfels CJ, Magnuson-Ford K, Barker MS, Rieseberg LH, et al. Recently formed polyploid plants diversify at lower rates. Science. 2011;333:1257.

22. Mayfield D, Chen ZJ, Pires JC. Epigenetic regulation of flowering time in polyploids. Curr Opin Plant Biol. 2011;14:174-8.

23. Nieto Feliner G, Álvarez I, Fuertes-Aguilar J, et al. Is homoploid hybrid speciation that rare? An empiricist's view. Heredity. 2017;118:513-6.

24. Otto SP. The evolutionary consequences of polyploidy. Cell. 2007;131:452-62.

25. Otto SP, Whitton J. Polyploid incidence and evolution. Annu Rev Genet. 2000;34:401-37.

26. Pandit MK, Pocock MJO, Kunin WE. Ploidy influences rarity and invasiveness in plants. J Ecol. 2011;99:1108-15.

27. Parisod C, Holderegger R, Brochmann C. (editors). Plant polyploidy. Evolutionary consequences of autopolyploidy. Special journal issue-New Phytol. 2010; 186(1).

28. Paule J, Wagner ND, Weising K, Zizka G. Ecological range shift in the polyploid members of the South American genus Fosterella (Bromeliaceae). Ann Bot. 2017;120:233-43.

29. Rice A, Šmarda P, Novosolov M, et al. The global biogeography of polyploid plants. Nat Ecol Evolut. 2019;3:265-73.

30. Stebbins GL. A brief summary of my ideas on evolution. Am J Bot. 1999;86:1207-8.

31. Stift M, Berenos C, Kuperus P, van Tienderen PH. Segregation models for disomic, tetrasomic and intermediate inheritance in tetraploids: a general procedure applied to Rorippa (yellow cress) microsatellite data. Genetics. 2008;179:2113-23.

32. Soltis DE, Soltis PS. Polyploidy: recurrent formation and genome evolution. Trends Ecol Evol. 1999;14:348-52.

33. Soltis DE, Soltis PS, Schemske DW, et al. Autopolyploidy in angiosperms: have we grossly underestimated the number of species? Taxon. 2007;56:13-30.

34. Soltis DE, Visger CJ, Marchant DB, Soltis PS. Polyploidy: pitfalls and paths to a paradigm. Am J Bot. 2016;103:1146-66.

35. Soltis DE, Visger CJ, Soltis PS. The polyploidy revolution then and now: Stebbins revisited. Amer J Bot. 2014;101:1057-78.

36. Soltis PS, Soltis DE. Polyploidy and genome evolution. Berlin: Springer; 2014. 
37. Soltis PS, Soltis DE. Ancient WGD events as drivers of key innovations in angiosperms. Curr Opin Plant Biol. 2016;30:159-65.

38. Spoelhof JP, Soltis PS, Soltis DE. Pure polyploidy: closing the gaps in autopolyploid research. J Syst Evol. 2017;55:340-52.

39. Stock M, Lamatsch DK. (eds). Special journal issue. Trends in polyploidy research in animals and plants. Cytogenet Genome Res 2013;40 (2 \&4).

40. Tate JA, Soltis DE, Soltis PS. Polyploidy in plants. In: Gregory TR, editor. The evolution of the genome. San Diego: Elsevier; 2005. p. 371-426

41. te Beest M, LeRoux JJ, Richardson DM, et al. The more the better? The role of polyploidy in facilitating plant invasions. Ann Bot. 2012;109:19-45.

42. Van de Peer Y, Maere S, Meyer A. The evolutionary significance of ancient genome duplications. Nat Rev Genet. 2009;10:725-32.

43. Van de Peer Y, Mizrachi E, Marchal K. The evolutionary significance of polyploidy. Nat Rev Genet. 2017;18:411-24. https://doi. org/10.1038/nrg.2017.26.
44. Vision TJ, Brown DG, Tanksley SD. The origins of genomic duplications in Arabidopsis. Science. 2000;290:2114-7.

45. Weiss-Schneeweiss H, Emadzade K, Jang TS, Schneeweiss GM. Evolutionary consequences, constraints and potential of polyploidy in plants. Cytogenet Genome Res. 2013;140:137-50.

46. Winkler $H$. Über die experimentelle erzeugung von pflantzen mitabweichenden chromosomenzahlen. Z Bot. 1916;8:417-531.

47. Wood TE, Takebayashi N, Barker MS, Mayrose I, Greenspoon PB, Rieseberg LH. The frequency of polyploid speciation in vascular plants. Proc Natl Acad Sci. 2009;106:13875-9.

Publisher's Note Springer Nature remains neutral with regard to jurisdictional claims in published maps and institutional affiliations. 